\title{
How Could I Pass, Why Should They Survive?
}

\author{
Ajay Malviya \\ Received: 23 July 2010 /Accepted: 17 November 2010 /Published online: 18 December 2010 \\ (C) Association of Surgeons of India 2010
}

\section{Dear Editor,}

I read with awe and interest the various articles that appear from time to time in various journals, many of which have found a place in standard textbooks.

Its not long ago that I appeared for my board examinations in general surgery. Twenty five years ago, the now almost obsolete clinical examination was been performed with great pains, investigations now relegated to history were still not readily available and treatments which cured or saved countless patients during my residency are now designated as not effective. Plain speakingly was it sheer quackery that I learned during my residency and passed my exams giving answers that are today considered outrightly wrong.

Flush ligation of cystic duct, an exhibition of surgical skill in those times is considered harmful to the patient and so is posterior oblique isoperistaltic gastrojejunostomy which is considered useless nowadays [1]. Is it that we are getting wiser today or that we were practising something which was not evidence based but pure conjectures that appealed to common sense which ultimately turned out to be sheer nonsense.

But what is most distressing is the fact that what amounted to be meticulous management in those days is considered unnecessary today. Masterly inactivity is the order of the day in the management of, be it peptic ulcer bleed or acute pancreatitis. While putting in a nasogastric tube and evaluating blood clots by saline washes be it cold or not so cold and ilk in cases of bleeding ulcer are laughed at and the only treatment advised is masterly inactivity after injecting a proton pump inhibitor [2]. Bleeding that we

A. Malviya $(\bowtie)$

Dr. S.N. Medical College,

30, Ashok Nagar, Link Pal Road,

Jodhpur Pin-342008 Rajasthan, India

e-mail: drmalviya.ajay@gmail.com thought stopped as a result of our painstaking endeavour in fact stopped by the 'GRACE OF GOD' to be correct.

Your present day 'gain' of knowledge leaves you with giving nothing in acute pancreatitis except intravenous fluids and play flute like Nero while the Rome burns or count various scores and pass time till he is relieved despite doing nothing i.e. by 'LUCK' or he passes away via an Intensive Care Unit.

Even present day liver abscesses have agreed to resolve with antibiotics and we have been communicated not to bother them by needles or drains.

Even drains, which although we were taught never drained,but were of great help in preventing and detecting complications are considered useless: may be because we are living in the era of tubeless tyres.

I wonder how could I pass when every correct answer that I gave for in our times has been proved wrong? But what intrigues me most is the fact that all those who survived shock syndrome by the use of Dopamine which had replaced her mother Noradrenaline only recently then (to our joy and excitement since so many patients were lost to Noradrenaline in those days) should have been dead since dopamine has now been condemned vis-a-vis noradrenaline which has regained the place of pride in the management of shock syndrome [3]. I don't understand why should all those have survived (..... our knowledge)?

\section{References}

1. Bailey and Love 17 th-25th editions

2. Farquharson's textbook of operative surgery 6 th- -9 th editions

3. Acute kidney injuries in critically ill: Lecture delivered by Dr. Rajshekhar Chakravarty at Criticare 2010 held at Hyderabad 10th14th Feb. 2010 